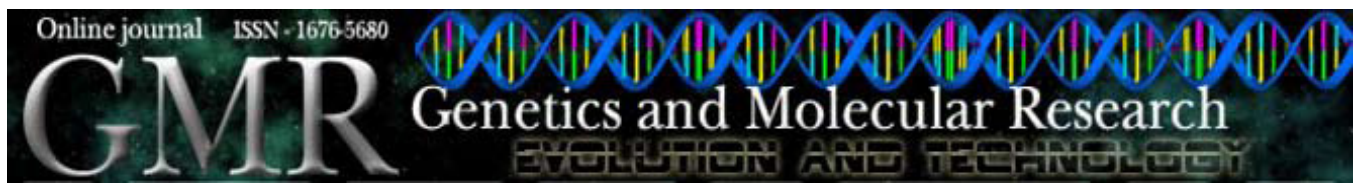

\title{
Correlation of aberrant expression of p53, Rad50, and cyclin-E proteins with microsatellite instability in gallbladder adenocarcinomas
}

\author{
P.K. Mishra ${ }^{1}$, S.K. Jatawa ${ }^{1}$, G.V. Raghuram ${ }^{1}$, N. Pathak ${ }^{1}$, A. Jain ${ }^{2}$, \\ A. Tiwari ${ }^{4}$, S. Varshney ${ }^{3}$ and K.K. Maudar ${ }^{3}$ \\ ${ }^{1}$ Department of Research and Training, \\ Bhopal Memorial Hospital and Research Centre, Bhopal, India \\ ${ }^{2}$ Department of Pathology, \\ Bhopal Memorial Hospital and Research Centre, Bhopal, India \\ ${ }^{3}$ Department of Surgical Gastroenterology, \\ Bhopal Memorial Hospital and Research Centre, Bhopal, India \\ ${ }^{4}$ School of Biotechnology, Rajiv Gandhi Technological University, \\ Bhopal, India
}

Corresponding author: P.K. Mishra

E-mail: pkm_8bh@yahoo.co.uk

Genet. Mol. Res. 8 (4): 1202-1210 (2009)

Received July 8, 2009

Accepted August 7, 2009

Published October 6, 2009

\begin{abstract}
Gallbladder carcinoma is an uncommon, but highly malignant tumor, with poor prognostic, and diagnostic manifestations in early stages. The Indian Council of Medical Research reported increased incidence of gallbladder carcinoma in the surviving population of the Bhopal gas tragedy that involved exposure of more than 500,000 people to methyl isocyanate gas. The severity of exposure, and increased multi-systemic morbidity in the survivors stimulated us to examine the molecular changes leading to gallbladder carcinoma. Surgically resected samples $(\mathrm{N}=40)$ of gallbladder carcinoma were studied for the p53, Rad50, and cyclin-E expression by immunohistofluorescence bioimaging. Among the 40 samples, 23, 11, and 10 showed p53, Rad50, and cyclin-E expression, respectively, in moderately differentiated adenocarcinomas, demonstrating the prevalence and inva-
\end{abstract}


siveness of this disease in the methyl isocyanate-exposed population $(\mathrm{P}=0.0009)$. Nevertheless, co-expression of Rad50, and cyclin-E with p53 was absent in adenomas with dysplasia, demonstrating their independent roles. We conclude that there was altered expression of $\mathrm{p} 53$, Rad50, and cyclin-E in the malignant transformation of gallbladder carcinoma in this methyl isocyanate gas-exposed cohort. Hence, these proteins may be useful as markers to identify premalignant lesions that are likely to progress into malignant adenocarcinoma.

Key words: Gallbladder cancer; Methyl isocyanate; p53; Rad50; Cyclin-E

\section{INTRODUCTION}

Gallbladder cancer is a common malignancy of the biliary tract, which shows wide geographical variation in its distribution. According to the American Cancer Society (2008) statistical projections, the number of new cases of gallbladder and other biliary cancers in the United States is predicted to be 4500 in men and 5020 in women (American Cancer Society, 2008). Areas with the highest incidence rates include Chile, India, Korea, Japan, Czech Republic, Slovakia, Spain, Columbia, Peru, Bolivia, and Ecuador (Randi et al., 2006). Females from India have the highest international rate of gallbladder cancer, between 8.8 and 21.2 per 100,000 persons/year, with high incidence of gallbladder cancer in north and central India (Kapoor and McMichael, 2003; Schottenfeld and Fraumeni, 2006).

Involuntary exposure to environmental carcinogens and xenochemicals could account for the recent growing incidence of exposure-related excess mortality due to gallbladder malignancies (Irigaray et al., 2007). However, the magnitude of risk fraction in gallbladder malignancies attributed to environmental factors is still incomprehensible. Global analysis of response of genes to toxic insult has been documented previously and provides a complete picture of toxicologically significant events (Crab, 2004). It has become increasingly evident that both kinds of exposures: accidents/disasters can potentially result in toxic genomic implications. The population-based cancer registry of the Indian Council of Medical Research (ICMR) revealed higher incidence rates (crude and age-adjusted incidence rates) of gallbladder carcinoma (GBC) in the surviving population of the Bhopal gas tragedy (Sriramachari, 2004). The tragedy is among the world's worst known industrial disasters, which led to the leakage of methyl isocyanate (MIC) and its related toxic gas products resulting in mortality of 2500-6000, and debilitating over 200,000 people. The severity of exposure was extreme, and the survivors continue to experience increased multi-systemic morbidity (Dhara and Dhara, 2002; Mishra et al., 2008; Mishra et al., 2009a), including cancer (Ganesh et al., 2005), which clearly establishes a genetic link with MIC exposure (Dikshit and Kanhere, 1999). The poor prognosis and high incidence rates of GBC in the MIC-exposed population further necessitate a better insight into the molecular changes to develop effective early diagnostics and novel targeted therapeutic strategies.

We aimed to study the expression of p53, cyclin-E, and Rad50 in gallbladder cancer specimens surgically resected from the MIC-affected population. The key feature of the p53 gene product (protein), "a guardian of the genome" with tumor suppressor function and a role in the regulation of cell cycle progression, is its tendency to remain "latent" in unperturbed 
cells (Horn and Vousden, 2007). Rad50 is a ubiquitous protein involved in many DNA metabolic pathways that maintain genomic integrity (Haber, 1998). Cyclin-E is one of the most important cell cycle regulators that play an important role in normal cell proliferation and development through promotion of S phases (Viallard et al., 2001). In this study, we used immunohistochemical analysis to determine the expression patterns of the $\mathrm{p} 53$, cyclin-E, and Rad50 proteins in 40 cases of GBC: 31 of adenocarcinoma, 6 of gallbladder adenoma with dysplasia and 3 of adenosquamous carcinoma.

\section{SUBJECTS AND METHODS}

The study was carried out by the Department of Research in coordination with the Department of Pathology, Bhopal Memorial Hospital and Research Centre (BMHRC), Bhopal, India, and patients from the Department of Surgical Gastroenterology formed the basis for the present research. The Institutional Review Board of BMHRC approved the study. Only subjects from 36 municipal wards considered "MIC affected" were selected for the study. Informed consent was obtained from each patient. Clinical details were recorded on pro forma charts in each case.

\section{Tissue specimens}

Tissue specimens from $40 \mathrm{GBC}$ patients ( 13 men and 27 women, age range of 20-75 years, mean age of $55.25 \pm 1.89$ years), including 31 with adenocarcinoma, 3 with adenosquamous carcinoma, and 6 with gallbladder adenoma, were examined immunohistochemically for $\mathrm{p} 53$, cyclin-E, and Rad50. A gallbladder adenoma was defined as any outgrowth that histologically showed gland proliferation and some grade of dysplasia. Gallbladder adenocarcinoma was defined as an outgrowth with cells showing atypicity, forming glands and originating on superficial epithelia that can be observed microscopically (Albores-Saavedra et al., 1992). All surgically resected specimens of gallbladder cancer were initially fixed in $10 \%$ neutralbuffered formalin, and then embedded in paraffin-wax after further dehydration processing. The cut sections were stained with hematoxylin and eosin. The diagnosis was based on clinical suspicion and histopathological confirmation in each patient.

\section{Immunohistochemical staining}

p53, cyclin-E, and Rad50 expression was examined by an immunohistofluorescence method using a spectral bio-imaging system (Applied Spectral Imaging, Germany). Briefly, 3- to 5- $\mu \mathrm{m}$ sections were cut from the paraffin embedded tissue blocks and placed on polyL-lysine coated slides. Immunohistochemcial staining of p53, cyclin-E, and Rad50 was performed using the indirect immunohistofluorescence method. In brief, the sections were deparaffinized with xylene rinse and rehydrated into distilled water through a graded alcohol series $(100,90$, and $70 \%)$. The mounted tissue was then rinsed with $1 \mathrm{X}$ phosphate-buffered saline on the slide. The slides were then incubated with primary mouse monoclonal anti-p53/antiRad50/anti-cyclin-E antibody (Santa Cruz Biotechnology Inc., USA) at a dilution of 1:1000 in a humidified chamber for $2-3 \mathrm{~h}$ at room temperature. The slides were washed three times in phosphate-buffered solution and further incubated with fluorescein isothiocyanate/Texas 
Red conjugated secondary antibody (Santa Cruz Biotechnology Inc.) at 1:40 dilution for $1 \mathrm{~h}$ at room temperature. Tissue nuclei were counterstained with DAPI (4,6-diamidinophenylindole), and incubated for 15-20 min. Finally, the slides were mounted with antifade solution and then examined using a spectral bio-imaging system (Applied Spectral Imaging).

\section{Criteria for positive immunohistochemical staining}

Results were quantitative and qualitative evaluations of immunohistochemical staining. p53, cyclin-E, and Rad50 immunoreactivity was considered to be positive when more than $10 \%$ of cells with nuclear, and membrane-bound or cytoplasmic staining were observed, respectively. Cytoplasmic staining for p53 was disregarded. Intensity of staining was graded as weak, moderate, and strong (Kim et al., 2001). Data were processed using Excel, and the SPSS 10.0 software (SPSS Inc., USA).

\section{Microsatellite instability}

Microsatellite instability was assessed using inter-simple sequence repeat polymerase chain reaction (ISSR-PCR), as reported elsewhere (Mishra et al., 2009b,c). Briefly, whole DNA was extracted from tissue specimens using the tissue protocol of DNeasy Blood \& Tissue kit (QIAGEN, Germany). An amount of $50 \mathrm{ng}$ of the extracted DNA was amplified, and analysis of the PCR product was performed by $1 \%$ agarose gel electrophoresis and imaging under a gel documentation system (Vilber Lourmat, France).

\section{RESULTS}

The 40 GBC cases in our study were of the following types: 27 (67.5\%) were females, and $13(32.5 \%)$ were males, and their histological classification was 31 adenocarcinoma, 3 adenosquamous carcinoma, and 6 adenoma with dysplasia (Table 1). We noted that p53 overexpression was predominantly seen in the MIC-exposed cohort diagnosed with adenocarcinoma, with 74\% (23/31) involving infiltration into the papillary, and mucinous region/cell types of the gallbladder (Table 1). In contrast, p53 expression was relatively moderate in adenosquamous carcinoma, and less in adenomas with dysplasia (Figure 1). According to the differentiation grade, gallbladder adenocarcinomas were further sub-grouped into well, moderately, and poorly differentiated adenocarcinomas. Although no significant correlation was shown between p53 overexpression, and differentiation grade, p53 overexpression was relatively stronger in the moderately differentiated adenocarcinoma cases when compared with moderate expression of well-differentiated cases (Table 2).

Table 1. Correlation of $\mathrm{p} 53$, Rad50 and cyclin-E over expression in gallbladder carcinoma.
\begin{tabular}{lcccc}
\hline Histological subtype & $\mathrm{p} 53$ & Rad50 & Cyclin-E & $\mathrm{P}$ \\
\hline Adenocarcinoma $(\mathrm{N}=31)$ & $23(74 \%)$ & $11(35 \%)$ & $10(32 \%)$ & 0.0009 \\
Adenosquamous carcinoma $(\mathrm{N}=3)$ & $1(33.3 \%)$ & $1(33.3 \%)$ & $0(0 \%)$ & $\mathrm{NS}$ \\
Adenomas with dysplasia $(\mathrm{N}=6)$ & $1(16.6 \%)$ & $0(0 \%)$ & $0(0 \%)$ & $\mathrm{NS}$ \\
\hline
\end{tabular}

Data are reported as number with percent in parentheses. NS = statistically non-significant. 


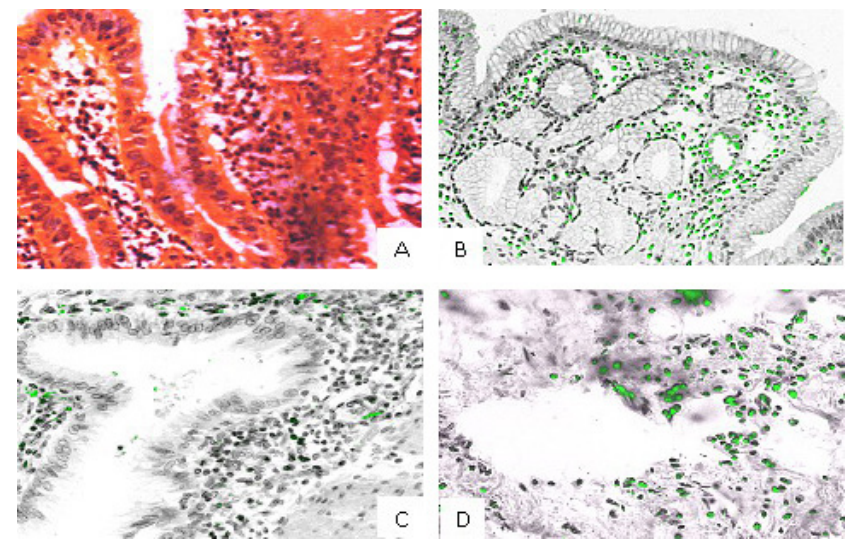

Figure 1. Over expression of p53 protein. Photomicrograph representing hematoxylin and eosin image of p53 overexpression (A), immunohistofluorescence detection of $\mathrm{p} 53$ protein overexpression in the nuclei of moderately differentiated adenocarcinoma (B), adenosquamous carcinoma (C), and adenoma with dysplasia (D) of gallbladder. (Green spots: positive nuclear staining; DAPI counter-stained tissue: grey color) (p53; original magnification 40X).

Table 2. Correlation of p53, Rad50 and cyclin-E over expression in adenocarcinomas of gallbladder based on differentiation grade.

\begin{tabular}{lrcc}
\hline Adenocarcinomas & $\mathrm{p} 53$ & Rad50 & Cyclin-E \\
\hline Differentiation grade $(\mathrm{N}=31)$ & & & \\
Well differentiated $(\mathrm{N}=7)$ & $4(57 \%)$ & $2(29 \%)$ & $1(14 \%)$ \\
Moderately differentiated $(\mathrm{N}=19)$ & $16(84 \%)$ & $9(52 \%)$ & $7(41 \%)$ \\
Poorly differentiated $(\mathrm{N}=5)$ & $2(40 \%)$ & $1(20 \%)$ & $1(20 \%)$ \\
\hline
\end{tabular}

Data are reported as number with percent in parentheses.

However, aberrant expression of Rad50 (Figure 2) was confined to adenocarcinoma and adenosquamous carcinoma with 11 (35\%), and 1 (33\%) cases, respectively (Table 1). Analysis of Rad50 expression based on differentiation grade displayed higher levels in moderately differentiated adenocarcinoma and lower levels in well and poorly differentiated adenocarcinoma (Table 2). However, Rad50 expression was not seen in adenomas with dysplasia.
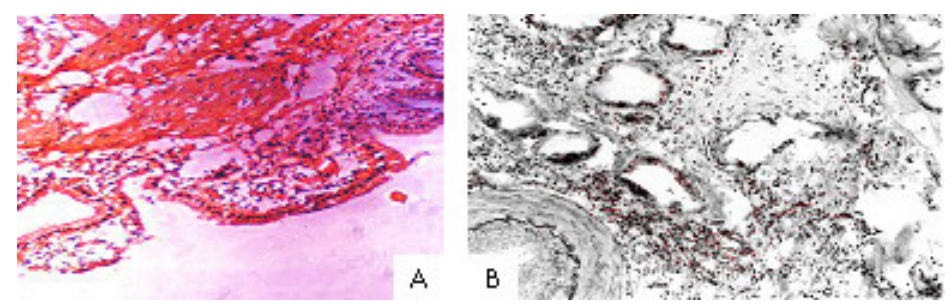

Figure 2. Photomicrograph showing hematoxylin and eosin staining (A) and immunostaining (red spots, B), strongly positive for aberrant expression of Rad50 protein in moderately differentiated adenocarcinoma of gallbladder. (Red spots: positive nuclear staining; DAPI counter-stained tissue: grey color) (Rad50; original magnification 20X). 
Meanwhile, anti-cyclin-E (Figure 3) showed positivity for cyclin-E overexpression only in adenocarcinoma of $\mathrm{GBC}$ with 10 cases (32\%), and stained none of the adenosquamous carcinoma and adenomas with dysplasia (Table 1). On the other hand, further assessment of adenocarcinoma revealed relatively stronger positive expression of cyclin-E in moderately differentiated adenocarcinoma than in poor, and well-differentiated adenocarcinomas (Table 2).
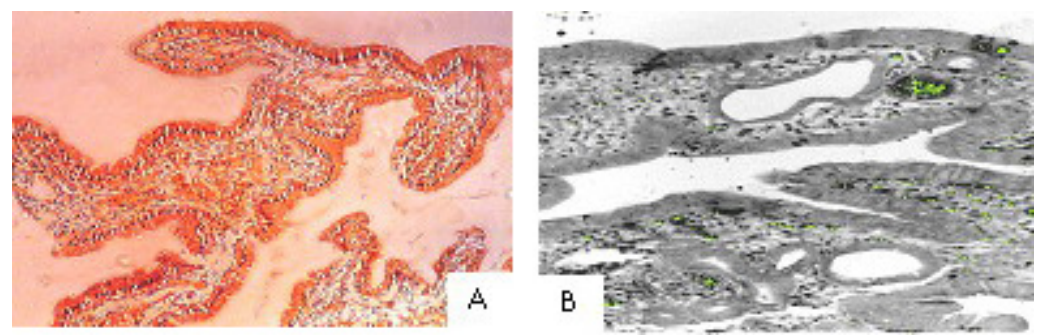

Figure 3. Photomicrograph showing moderately differentiated adenocarcinoma, hematoxylin and eosin stained (A), immunopositive for cyclin-E protein over expression (B) in gallbladder. (Green spots: positive nuclear staining in background of DAPI counter-stained tissue: grey color) (Cyclin-E; original magnification 20X).

ISSR-PCR analysis did not demonstrate any significant microsatellite instability in the GBC cases studied (data not shown).

\section{DISCUSSION}

Carcinogenesis is a multistep and age-dependent process, and environmental factors are thought to play an important role in it. Genetic susceptibility to cancer due to genetic polymorphism favors the role of exogenous factors through gene-environment interactions (Schottenfeld and Fraumeni, 2006). In GBC, little is known about the molecular mechanisms underlying tumor progression and metastasis, yet it results in fatal malignancies. The poor prognosis associated with GBC is due to its inherent aggressive biology and diagnosis at the advanced stage and anatomic position with vague and non-specific symptoms, along with a poor survival rate of less than $5 \%$ (Saetta et al., 2006). Considerable variation exists in the incidence and mortality rates of gallbladder cancer throughout the world, which suggests that genetic and environmental variables may be involved in its tumorigenesis. Of these, gallstones and chronic inflammation of the gallbladder are the most common etiological factors (Pandey, 2003). However, attempts have been made to determine the evidence on the role of occupational factors and environmental mutagens in gallbladder carcinogenesis. In this regard, some studies have shown the possibility of carcinogen involvement in the events leading to GBC. Increased risk for gallbladder malignancies among workers in the oil, paper, pesticides, rubber, chemical, and textile industries suggests a role of industrial chemicals in carcinogenesis (Szymczak et al., 2003; Kuzmickiene et al., 2004; Kumar et al., 2006).

The role of various oncogenic mutations in gallbladder cancer is an area of active research since no promising biomarker has been found that can distinguish GBC at an early stage. This provided the rationale for us to evaluate the status of the most commonly disrupted genes, namely p53, Rad50, and cyclin-E. Very few reports in the literature are available on the 
study of p53 in gallbladder cancer in Indian patients (Misra et al., 2000), and the role of cyclin$\mathrm{E}$ as prognosticator in gastric cancers is debatable. Also, there is no information available on Rad50 expression in gallbladder cancer to date.

Protein p53 is a regulator of the cell cycle and one of the most frequently altered targets in the majority of human neoplasias. Its main function is to maintain the integrity of DNA and induce apoptosis of those cells with an abnormal DNA impossible to be repaired. It has been shown that mutated p53 is involved in the pathogenesis of carcinoma of the gallbladder, and appears to be one of the factors involved in the genesis of this process (da Rocha et al., 2004). Aberrant expression of $\mathrm{p} 53$ in the gallbladder has been reported mainly in adenocarcinomas (35-65\%) (Roa et al., 2000), but it has also been found in adenomas (0-17\%) (Wang et al., 2006). Our results are in accordance with those reported in the literature, since we found p53 overexpression in $74 \%$ of the adenocarcinomas and only in $16.6 \%$ of the adenomas with dysplasia. This variable expression of p53 (Figure 1) further suggests that adenomas express p53 very late in their development to adenocarcinoma and also that the molecular events and origins of p53 are different in adenocarcinomas and adenomas (Wistuba et al., 1999).

Rad50 is a highly conserved DNA double-strand break repair factor. This protein complex integrates DNA repair with checkpoint signaling, downstream in the DNA damage response pathway (Lee and Paull, 2004). Cyclin-E protein controls the cell cycle, allowing progression from $\mathrm{G} 1$ to $\mathrm{S}$ phase. Cyclin-E alteration such as untimely and aberrant expression can cause deregulation of the cell cycle by speeding up the G1 phase and thus contributes to carcinogenesis and malignant transformation through subsequent unbridled cell growth and proliferation. Thus, it is natural that its overexpression leads to cell proliferation found in a variety of human cancers. Overexpression of cyclin-E has been reported to occur in gastric cancer, making it a potential prognostic marker (Jang et al., 1999; Aoyagi et al., 2000). The present study also demonstrated the expression of Rad50 (35\%), and cyclin-E $(32 \%)$ in adenocarcinomas but showed statistically significant $(\mathrm{P}=0.0009)$ difference in expression pattern compared to p53 (Table 1).

Moreover, a direct correlation exists between the presence of cholelithiasis/cholecystitis, and the development of gallbladder carcinoma. In patients with gallbladder carcinoma, the incidence of cholelithiasis ranges from 54 to $97 \%$ with the most common histological type being "adenocarcinoma", while others such as undifferentiated or anaplastic carcinoma, squamous cell carcinoma, and adenosquamous carcinoma represent less common types of gallbladder cancers (Bhuta et al., 2002). In the present study, our results support those reported in the literature, since we found the presence of cholelithiasis and acute/chronic cholecystitis in the cohort that was diagnosed with adenocarcinomas. Notably, the overexpression of p53, Rad50, and cyclin-E was seen in moderately differentiated adenocarcinoma as depicted by the infiltration of adenocarcinoma into papillary, tubular, and mucinous part of gallbladder tissue (Figures 1-3). Furthermore, the results of the Rad50, and cyclin-E expression selectively in adenocarcinomas signify the evident mutations occurring in these genes, and their potential role in malignant transformation of GBC along with the pertinent p53 expression observed in the study (Figures 1-3). The moderate co-expressions of Rad50, and negligible expression of cyclin-E in adenosquamous and adenomas with dysplasia in comparison to p53 respectively indicate their little importance in the development of adenosquamous, and adenomas of the gallbladder, and Rad50, and cyclin-E may have an independent role in the carcinogenesis of the gallbladder. Thus, substantial expression of $\mathrm{p} 53$, and variable expression of $\operatorname{Rad} 50$, and 
cyclin-E in the exposed population diagnosed with adenocarcinomas, adenosquamous carcinoma, and adenomas with dysplasia suggest their role in the carcinogenesis of the gallbladder.

Microsatellite instability, which represents replication errors, results from DNA mismatches due to environmental or hereditary factors, and leads to genomic instability (Peltomaki, 1997; Jackson et al., 1998). The reported prevalence of high microsatellite instability for sporadic gallbladder carcinoma ranges from 0 to 10\% (Yoshida et al., 2000; Rashid et al., 2002; Yanagisawa et al., 2003; Roa et al., 2005); correspondingly, in our cases, the prevalence was negligible (data not shown). This suggests that microsatellite instability plays a minor role in the pathogenesis of gallbladder cancer cases in the present study.

To conclude, our study is the first of its kind to reveal the expression patterns of $\mathrm{p} 53$, Rad50, and cyclin-E in an MIC-exposed population diagnosed with gallbladder cancer, thereby revealing the prevalence of invasive carcinoma of the gallbladder in the cohort studied. Analogously, the study herein also demonstrated the aggressiveness of the disease in the central Indian population as reported earlier in the literature (Kapoor and McMichael, 2003). However, a larger sample size would validate the results and efficacy of these proteins in developing effective early diagnostics and novel targeted therapeutic strategies against cancers of the gallbladder.

\section{ACKNOWLEDGMENTS}

The authors are thankful to Bhopal Memorial Hospital Trust, India, for providing the necessary financial support and Ms. K. Parveen and Ms. V. Bhatia for necessary technical assistance.

\section{REFERENCES}

Albores-Saavedra J, Henson DE and Sobin LH (1992). The WHO histological classification of tumors of the gallbladder and extrahepatic bile ducts. A commentary on the second edition. Cancer 70: 410-414.

American Cancer Society (2008). Key Statistics for Gallbladder Cancer. Available at [http://www.cancer.org/docroot/CRI/content/CRI_2_4_1X_What_are_the_key_statistics_for_gall_bladder_cancer_68.asp?sitearea=]. Accessed March 29, 2008.

Aoyagi K, Koufuji K, Yano S, Murakami N, et al. (2000). Immunohistochemical study on the expression of cyclin D1 and E in gastric cancer. Kurume Med. J. 47: 199-203.

Bhuta P, Brown MG and Alderdice JM (2002). Gall bladder cancer - radical surgery, the key role to improve outcome. Ulster Med. J. 71: 128-131.

Crabb C (2004). Revisiting the Bhopal tragedy. Science 306: 1670-1671.

da Rocha AO, Coutinho LM, Scholl JG and Leboutte LD (2004). The value of p53 protein expression in gallbladder carcinoma: analysis of 60 cases. Hepatogastroenterology 51: 1310-1314.

Dhara VR and Dhara R (2002). The Union Carbide disaster in Bhopal: a review of health effects. Arch. Environ. Health 57: 391-404.

Dikshit RP and Kanhere S (1999). Cancer patterns of lung, oropharynx and oral cavity cancer in relation to gas exposure at Bhopal. Cancer Causes Control 10: 627-636.

Ganesh N, Sanyal B, Panday RK, Patel AK, et al. (2005). Cancer pattern among MIC gas survivor and their offspring. Health Administrator 17: 50-58.

Haber JE (1998). The many interfaces of Mre11. Cell 95: 583-586.

Horn HF and Vousden KH (2007). Coping with stress: multiple ways to activate p53. Oncogene 26: 1306-1316.

Irigaray P, Newby JA, Clapp R, Hardell L, et al. (2007). Lifestyle-related factors and environmental agents causing cancer: an overview. Biomed. Pharmacother. 61: 640-658.

Jackson AL, Chen R and Loeb LA (1998). Induction of microsatellite instability by oxidative DNA damage. Proc. Natl. Acad. Sci. U. S. A. 95: 12468-12473. 
Jang SJ, Park YW, Park MH, Lee JD, et al. (1999). Expression of cell-cycle regulators, cyclin E and p21WAF1/CIP1, potential prognostic markers for gastric cancer. Eur. J. Surg. Oncol. 25: 157-163.

Kapoor VK and McMichael AJ (2003). Gallbladder cancer: an 'Indian' disease. Natl. Med. J. India 16: 209-213.

Kim YW, Huh SH, Park YK, Yoon TY, et al. (2001). Expression of the c-erb-B2 and p53 protein in gallbladder carcinomas. Oncol. Rep. 8: 1127-1132.

Kumar S, Kumar S and Kumar S (2006). Infection as a risk factor for gallbladder cancer. J. Surg. Oncol. 93: 633-639.

Kuzmickiene I, Didziapetris R and Stukonis M (2004). Cancer incidence in the workers cohort of textile manufacturing factory in Alytus, Lithuania. J. Occup. Environ. Med. 46: 147-153.

Lee JH and Paull TT (2004). Direct activation of the ATM protein kinase by the Mre11/Rad50/Nbs1 complex. Science 304: 93-96.

Mishra PK, Gupta D, Chauhan C, Panwar H, et al. (2008). Status of Medical Biotechnology Research in the State of Madhya Pradesh: Past Accomplishments Current Trends and Challenges Ahead. In: A Review of Long Term Health Effects amongst the Survivors of Bhopal Gas Tragedy (Mishra PK and Maudar KK, eds.). BMHRC (Bhopal Memorial Hospital and Research Centre) Publication, Bhopal, 73-90.

Mishra PK, Dabadghao S, Modi GK, Desikan P, et al. (2009a). In utero exposure to methyl isocyanate in the Bhopal gas disaster: evidence of persisting hyperactivation of immune system two decades later. Occup. Environ. Med. 66: 279.

Mishra PK, Bhargava A, Raghuram GV, Gupta S, et al. (2009b). Inflammatory response to isocyanates and onset of genomic instability in cultured human lung fibroblasts. Genet. Mol. Res. 8: 129-143.

Mishra PK, Bhargava A, Raghuram GV, Jatawa SK, et al. (2009c). Induction of genomic instability in cultured human colon epithelial cells following exposure to isocyanates. Cell Biol. Int. 33: 675-683.

Misra S, Chaturvedi A, Goel MM, Mehrotra R, et al. (2000). Overexpression of p53 protein in gallbladder carcinoma in North India. Eur. J. Surg. Oncol. 26: 164-167.

Pandey M (2003). Risk factors for gallbladder cancer: a reappraisal. Eur. J. Cancer Prev. 12: 15-24.

Peltomaki P (1997). DNA mismatch repair gene mutations in human cancer. Environ. Health Perspect. 105 (Suppl 4): 775-780.

Randi G, Franceschi S and La Vecchia C (2006). Gallbladder cancer worldwide: geographical distribution and risk factors. Int. J. Cancer 118: 1591-1602.

Rashid A, Ueki T, Gao YT, Houlihan PS, et al. (2002). K-ras mutation, p53 overexpression, and microsatellite instability in biliary tract cancers: a population-based study in China. Clin. Cancer Res. 8: 3156-3163.

Roa I, Melo A, Roa J, Araya J, et al. (2000). P53 gene mutation in gallbladder cancer. Rev. Med. Chil. 128: 251-258.

Roa JC, Roa I, Correa P, Vo Q, et al. (2005). Microsatellite instability in preneoplastic and neoplastic lesions of the gallbladder. J. Gastroenterol. 40: 79-86.

Saetta AA, Gigelou F, Papanastasiou PI, Koilakou SV, et al. (2006). High-level microsatellite instability is not involved in gallbladder carcinogenesis. Exp. Mol. Pathol. 80: 67-71.

Schottenfeld D and Fraumeni J (2006). Cancer Epidemiology and Prevention. 3rd edn. Oxford University Press, New York.

Sriramachari S (2004). Cancer Patterns in MIC/Toxic Gas Affected and Un-Affected Areas of Bhopal (1988-2003). In: Health Effects of the Toxic Gas Leak from the Union Carbide Methyl Isocyanate Plant in Bhopal Indian Council for Medical Research, New Delhi, 171-177.

Szymczak W, Sobala W, Wilczynska U and Szeszenia-Dabrowska N (2003). Assessment of risk of death due to malignant neoplasms induced by occupational exposure in a rubber footwear plant. Med. Pr. 54: 221-228.

Viallard JF, Lacombe F, Belloc F, Pellegrin JL, et al. (2001). Molecular mechanisms controlling the cell cycle: fundamental aspects and implications for oncology. Cancer Radiother. 5: 109-129.

Wang SN, Chung SC, Tsai KB, Chai CY, et al. (2006). Aberrant p53 expression and the development of gallbladder carcinoma and adenoma. Kaohsiung. J. Med. Sci. 22: 53-59.

Wistuba II, Miquel JF, Gazdar AF and Albores-Saavedra J (1999). Gallbladder adenomas have molecular abnormalities different from those present in gallbladder carcinomas. Hum. Pathol. 30: 21-25.

Yanagisawa N, Mikami T, Yamashita K and Okayasu I (2003). Microsatellite instability in chronic cholecystitis is indicative of an early stage in gallbladder carcinogenesis. Am. J. Clin. Pathol. 120: 413-417.

Yoshida T, Sugai T, Habano W, Nakamura S, et al. (2000). Microsatellite instability in gallbladder carcinoma: two independent genetic pathways of gallbladder carcinogenesis. J. Gastroenterol. 35: 768-774. 